\title{
Inventory and Pricing Decisions Under Wholesale Price Contract with Social Preferences
}

\author{
Shuren LIU \\ School of Mathematics and Computational Science, Xiangtan University, Xiangtan 411105, China; \\ Business School, Xiangtan University, Xiangtan 411105, China \\ E-mail:liushuren@xtu.edu.cn \\ Huina CHEN \\ School of Mathematics and Computational Science, Xiangtan University, Xiangtan 411105, China \\ E-mail: 1617160665@qq.com \\ Lili CHEN \\ School of Mathematics and Computational Science, Xiangtan University, Xiangtan 411105, China \\ E-mail:772368343@qq.com
}

\begin{abstract}
This paper introduces the other-regarding preferences coefficients and studies the impact of social preferences on supply chain performance in the price-setting newsvendor setting. It is assumed that the stochastic demand is multiplicative. The manufacturer and retailer play a Stackelberg game. We analyze the impact of the decision-maker's social preferences on the manufacturer's optimal wholesale price, the retailer's optimal retail price and order quantity, the supply chain member's profits and utilities, and the supply chain system's profits and utilities under three different cases that only the retailer, only the manufacturer and both are with social preferences. We show that a manufacturer, as a leader, should find a spiteful retailer, while a retailer, as a follower, should find a manufacturer with generous liability, to improve the entire supply chain. Finally, numerical examples are given to illustrate these results.
\end{abstract}

Keywords social preference; supply chain management; wholesale price contract; price-setting newsvendor

\section{Introduction}

A great deal of attention has been paid to supply chain management for over a decade, where supply chain coordination is mainly discussed due to double marginalization in decentralized chains. There is a good deal of analytical modelling literature in operating, starting with Spengler ${ }^{[1]}$, that deals with designing contracts to align incentives (Cachon ${ }^{[2]}$ ). However, although the wholesale price contract can not coordinate the supply chain, we focus our analysis on it because it is heavily used in practice and, in fact, may be preferred over more complex contracts due to bounded rationality (Kalkanci, et al. ${ }^{[3,4]}$ ). Furthermore, most supply chain

Received September 10, 2015, accepted November 10, 2015

Supported by National Natural Science Foundation of China (71401150) 
contracting models are based on the assumption of self-interested, rational agents and exclude social considerations, such as reciprocity, fairness, and status seeking. However, recent developments in behavioral economics suggest that decision makers may care about reciprocity, fairness, and status in addition to economic benefits (see, for example, Rabin ${ }^{[5]}$, Fehr and Schmidt ${ }^{[6]}$, Charness and Rabin ${ }^{[7]}$, Loch and $\mathrm{Wu}^{[8]}$, Urda and $\operatorname{Loch}^{[9]}$ ). Our work serves to contribute to the recent stream of literature incorporating social preferences into decision making and supply chain interactions ${ }^{[10-12]}$. Based on the price-setting newsvendor model, this paper studies the coexistence of competition and cooperation in a two-echelon supply chain with wholesale price contract where pricing and ordering decisions are considered simultaneously. On the other hand, we introduce social preferences into decision maker's utility to study the coopetition in the supply chain.

Fairness has been long recognized as one of the most important factors guiding human interactions in everyday life. It is closely related to the other-regarding preferences, such as status, altruism, reciprocity, so common in the everyday life of individuals, which also plays an important role in the corporate environment. Liu, et al. ${ }^{[13]}$ identified four dimensions of fairness relevant in supplier-buyer relationships: Distributional, procedural, interpersonal, and informational. On the distributional aspect of fairness, Fehr and Schmidt ${ }^{[6]}$ defined fairness as a $50 / 50$ split of profits to study behavior in the fixed-pie ultimatum and dictator games. Decisions which result in advantageous allocations incur disutility with one rate, while disadvantageous allocations incur disutility at a higher rate. Ideal allocation reference points on the fairness preference of inequity aversion are 50/50 or more generally $\frac{\lambda}{1-\lambda}(0<\lambda<1)$. Later, Charness and Rabin ${ }^{[7]}$ generalize the preferences to allow for social welfare or competitive preferences (i.e., a player's utility is always increasing or decreasing in the other's payoff). That is to say, they extend ideal allocations to all or nothing (i.e., $\lambda=0$ or $\lambda=1$ ). Furthermore, Loch and $\mathrm{Wu}^{[8]}$ provided experimental evidence that social welfare or competitive preferences systematically affect economic decision making in supply chain transactions. Based on the price-setting newsvendor model, we explore the coopetition of supply chain players with social welfare or competitive preferences similar to [8], which has not been studied yet in the literature.

Based on [6], Cui, et al. ${ }^{[10]}$ first modeled fairness concerns in the context of channel coordination. They develop a model where both parties care about fairness in a bilateral monopoly setting with a supplier and a retailer, and find that the supplier can coordinate the channel using wholesale price contract under sufficient concerns for fairness and linear price-sensitive demand. Demirag, et al. ${ }^{[14]}$ extended [10] to other nonlinear demand functions that are commonly used in the literature, and reveal that the exponential demand function requires less stringent conditions to achieve coordination when only the retailer is fairness-concerned. Katok, et al. ${ }^{[15]}$ extended the model to include incomplete information on the fairness preference coefficients. Ding, et al. ${ }^{[16]}$ extended the model to a quantity discount mechanism, and show that channel coordination can be achieved by setting a simple wholesale price and fixed cost as long as the degree of attention of retailer to supplier's profit and the retailer's fairness preference coefficients satisfy certain conditions. Yang, et al. ${ }^{[17]}$ studied cooperative advertising in a distribution channel with fairness concerns and show that channel coordination can be achieved if the retailer has fairness concerns and model parameters satisfy certain condition. Wu and 
Niederhoff ${ }^{[18]}$ reformulated the existing inequity averse utility function as a piecewise function with clearly comparable parameters and study the impact of fairness concerns on supply chain performance in the newsvendor setting. Recently, Ho, et al. ${ }^{[19]}$ studied a model with a supplier and two-retailer supply chain setting, and investigate the interaction between distributional and peer-induced fairness. On the other hand, by social welfare or competitive preferences proposed in [7-8], Du, et al. ${ }^{[11]}$ discussed how the retailer's behavior of competitive preferences influences the coordination of supply chain, including the wholesale price contract, the buyback contract and the revenue-sharing contract. They find that the retailer's behavior of competitive preferences don't change the state of supply chain coordination. $\mathrm{Ge}$ and $\mathrm{Hu}^{[20]}$ studied the firms' altruistic incentives in a supply chain, and showed that the performance in the supply chain in consideration of altruism is between those of scenarios under decentralization and under integration. Shi, et al. ${ }^{[21]}$ studied the effect of altruism on retailer's and manufacturer's pricing strategy in two classic dual-channel supply chains by presenting Stackelberg game models.

In this paper, we introduce the other-regarding preferences coefficients and study the impact of social preferences on the inventory and pricing decisions in a two-echelon supply chain with wholesale price contract. It is assumed that the stochastic demand is multiplicative. The manufacturer and retailer play a Stackelberg game. We analyze the impact of the decision maker's social preferences on the manufacturer's optimal wholesale price, the retailer's optimal retail price and order quantity, the supply chain member's profits and utilities, and the supply chain system's profits and utilities under three different cases that only the retailer, only the manufacturer and both are with social preferences. We show that a manufacturer, as a leader, should find a spiteful retailer, while a retailer, as a follower, should find a manufacturer with generous liability, to improve the entire supply chain. Finally, numerical examples are given to illustrate these results.

The paper is organized as follows. Section 2 establishes two models of decision-making channel under traditional case. One is the integrated channel and the other is independent channel. Section 3 studies a supply chain selling to the price-setting newsvendor with social preferences. Section 4 gives some numerical examples, and Section 5 is the conclusion.

\section{The Integrated Channel and Independent Channel}

In order to facilitate the research, we consider a simple distribution channel consisting of one manufacturer and one retailer. The manufacturer is an upstream monopolist in the distributional channel and the retailer is a monopolist seller in the consumer market. The manufacturer and retailer play a Stackelberg game. The manufacturer produces a product at a marginal cost $c$ and offers a wholesale price $w$ to the retailer. The retailer determines the retail price $p$ and order quantity $q$, and suffers no additional selling costs or charges except the wholesale price. Assume that stochastic demand for the product has the following multiplicative functional form:

$$
D(p, \varepsilon)=y(p) \varepsilon,
$$

where $y(p)$ is a deterministic and decreasing function of the product's selling price $p$, and $\varepsilon$ is a random factor with distribution function $F($.$) , density function f($.$) and a mean value of \mu$. It is assumed that the probability distribution has support on $[A, B]$ with $B>A \geq 0$ and so $\mu>0$. 
We let $y(p)$ take the form of $y(p)=a p^{-b}$, where $a>0$ and $b>1$. The demand function form is one of the few models that have often been adopted in the literature for studying joint pricinginventory management in supply chain context (see, for example, Petruzzi and Dada ${ }^{[22]}$, Wang, et al. ${ }^{[23]}$, Song, et al. ${ }^{[24]}$ ). In this formulation, the parameter $b$ is the price-elasticity index of demand. The larger the value of $b$ is, the more sensitive the demand is to a change in price. If the price-elasticity index is 1 or less, then a product is defined as price-inelastic; Otherwise, a product is defined as price-elastic. We focus on price-elastic products by assuming $b>1$.

Before proceeding to the detailed analysis, we present the following mild assumption about $\varepsilon$. As in [25], we define $g(x)=x h(x)$ as the generalized failure rate of the demand distribution for $\varepsilon$, where $h(x)=\frac{f(x)}{1-F(x)}$ is the failure rate function.

Assumption $1 g(x)$ is increasing for $x \in[A, B]$.

The above increasing generalized failure rate (IGFR) property of $\varepsilon$ is indeed satisfied by most of the theoretical distribution used in the literature (see [24] and [25] for details).

\subsection{The Integrated Channel}

As the baseline of comparison, we first analyze the distribution channel decisions. If the distribution channel is integrated, the manufacturer and the retailer cooperate to maximize the profit of channel. Let $\Pi_{I}(p, q)$ denote the expected channel profit for any chosen price $p$ and production quantity $q$. We have

$$
\Pi_{I}(p, q)=p E[\min \{q, D(p, \varepsilon)\}]-c q=p E[\min \{q, y(p) \varepsilon\}]-c q .
$$

As in [22], we define $z=\frac{q}{y(p)}$, and call it the stocking factor of inventory. Then, the problem of choosing a price $p$ and production quantity $q$ is equivalent to choosing a price $p$ and a stocking factor $z$. Due to $E[\min \{z, \varepsilon\}]=z-\int_{A}^{z}(z-x) f(x) \mathrm{d} x=z-\Lambda(z)$, where $\Lambda(z)=\int_{A}^{z}(z-x) f(x) \mathrm{d} x$, the object function (1) can be written as

$$
\Pi_{I}(p, z)=p y(p) E[\min \{z, \varepsilon\}]-c q=y(p)\{p[z-\Lambda(z)]-z c\}=a p^{-b}\{p[z-\Lambda(z)]-z c\} .
$$

For any fixed $z$, it follows from (2) that

$$
\frac{\partial \Pi_{I}(p, z)}{\partial p}=a p^{-(b+1)}\{b c z-(b-1)[z-\Lambda(z)] p\} .
$$

Let $\frac{\partial \Pi_{I}(p, z)}{\partial p}=0$, then

$$
p^{I}(z)=\frac{b c z}{(b-1)[z-\Lambda(z)]}
$$

Note that $a p^{-(b+1)}>0$ due to $a>0$. Let $G(p)=b c z-(b-1)[z-\Lambda(z)] p$, then $G^{\prime}(p)=$ $-(b-1)[z-\Lambda(z)]<0$, so $G(p)$ is decreasing in $p$. Therefore, if $p>p^{I}(z)$, we have $G(p)<$ $G\left(p^{I}(z)\right)=0$, which indicates that $\frac{\partial \Pi_{I}(p, z)}{\partial p}<0$; If $p<p^{I}(z)$, we have $G(p)>G\left(p^{I}(z)\right)=0$, which indicates that $\frac{\partial \Pi_{I}(p, z)}{\partial p}>0$. Thus, $p^{I}(z)$ is the unique maximum point of $\Pi_{I}(p, z)$ for any fixed $z$.

By substituting (3) into (2), we have by the chain rule

$$
\begin{aligned}
\frac{\mathrm{d} \Pi_{I}\left(p^{I}(z), z\right)}{\mathrm{d} z} & =\frac{\partial \Pi_{I}\left(p^{I}(z), z\right)}{\partial z}+\frac{\partial \Pi_{I}\left(p^{I}(z), z\right)}{\partial p} \cdot \frac{\mathrm{d} p^{I}(z)}{\mathrm{d} z} \\
& =a\left[p^{I}(z)\right]^{-b}\left\{p^{I}(z)[1-F(z)]-c\right\}
\end{aligned}
$$




$$
=\frac{a c\left[p^{I}(z)\right]^{-b}}{(b-1)[z-\Lambda(z)]}\{z-b z F(z)+(b-1) \Lambda(z)\},
$$

where we use the fact that $\frac{\partial \Pi_{I}\left(p^{I}(z), z\right)}{\partial p}=0$ due to the optimality of $p^{I}(z)$. Note that

$$
\frac{a c\left[p^{I}(z)\right]^{-b}}{(b-1)[z-\Lambda(z)]}>0
$$

in (4). Let $H(z)=z-b z F(z)+(b-1) \Lambda(z)$, then $H^{\prime}(z)=[1-F(z)][1-b z h(z)], H^{\prime \prime}(z)=$ $-h(z) H^{\prime}(z)-b[1-F(z)]\left[h(z)+z h^{\prime}(z)\right]$. From Assumption 1, we have $g^{\prime}(z)=h(z)+z h^{\prime}(z)>0$, then $H^{\prime \prime}(z)<0$ at $H^{\prime}(z)=0$, which implies that $H(z)$ is a unimodal function. On the other hand, it is easy to see that $H(A)=A>0, H(B)=-(b-1) \mu<0$. Thus, we obtain an unique $z^{I}$ in the interval $[A, B]$. Therefore, from $H(z)=0$, we have

$$
F\left(z^{I}\right)=\frac{z^{I}+(b-1) \Lambda\left(z^{I}\right)}{b z^{I}} .
$$

In summary, we have the following optimal retail price $p^{I}$ and production quantity $q^{I}$ under the integrated channel case:

$$
\left\{\begin{array}{l}
F\left(z^{I}\right)=\frac{z^{I}+(b-1) \Lambda\left(z^{I}\right)}{b z^{I}} \\
p^{I}(z)=\frac{b c z}{(b-1)[z-\Lambda(z)]} \\
q^{I}(z)=a p^{-b} z
\end{array}\right.
$$

\subsection{The Independent Channel}

If the channel members are independent, the manufacturer and the retailer play a Stackelberg game. The manufacturer first offers a wholesale price $w$. According to the wholesale price, if the retailer rejects the sale contract, it will lead to zero profits, and the game is over. However, if the retailer accepts the offer, he or she will determine the order quantity $q$ and retail price $p$ to maximize his or her profits according to the given wholesale price $w$. Under the independent channel case, the retailer's problem is analogous to the integrated channel's expected profit except that he or she orders goods at price $w$ instead of producing it at price $c$. His or her expected profit is

$$
\Pi_{r}(p, q)=p E[\min \{q, D(p, \varepsilon)\}]-w q=p E[\min \{q, y(p) \varepsilon\}]-w q .
$$

Similar to the analysis under the integrated channel case, the profit function (7) can be written as

$$
\Pi_{r}(p, z)=p y(p) E[\min \{z, \varepsilon\}]-w q=y(p)\{p[z-\Lambda(z)]-z w\}=a p^{-b}\{p[z-\Lambda(z)]-z w\} .
$$

Further, we can obtain $p^{0}(z)=\frac{b w z}{(b-1)[z-\Lambda(z)]}$ and $F\left(z^{0}\right)=\frac{z^{0}+(b-1) \Lambda\left(z^{0}\right)}{b z^{0}}$.

Denoting the manufacturer's profit function by $\Pi_{m}(w)$, we have

$$
\Pi_{m}(w)=(w-c) q=(w-c) z y(p)=a p^{-b} z(w-c) .
$$

By substituting $p^{0}(z)=\frac{b w z}{(b-1)[z-\Lambda(z)]}$ into (9), we have

$$
\Pi_{m}(w)=\frac{a(b-1)^{b}[z-\Lambda(z)]^{b}}{b^{b} z^{b-1}} \cdot I(w),
$$


where

$$
I(w)=(w-c) w^{-b} .
$$

From $F\left(z^{0}\right)=\frac{z^{0}+(b-1) \Lambda\left(z^{0}\right)}{b z^{0}}$, we can conclude that $z^{0}$ is independent of $w$. Thus, maximizing $\Pi_{m}(w)$ over $w$ is equivalent to maximizing the function $I(w)$. By (10), we have

$$
\frac{\partial I(w)}{\partial w}=w^{-(b+1)}[w-b(w-c)] .
$$

Let $\frac{\partial I(w)}{\partial w}=0$, then $w^{0}=\frac{b}{b-1} c$. Further, let $J(w)=w-b(w-c)$, then $J^{\prime}(w)=1-b<0$ for $b>1$, so $J(w)$ is decreasing in $w$. Note that $w^{-(b+1)}>0$. Thus, if $w>w^{0}$, we have $\frac{\partial I(w)}{\partial w}<0$; if $w<w^{0}$, we have $\frac{\partial I(w)}{\partial w}>0$. So, $w^{0}=\frac{b}{b-1} c$ is the unique maximum point of $I(w)$.

In summary, we have the following optimal wholesale price $w^{0}$ for the manufacturer, the optimal retail price $p^{0}$ and order quantity $q^{0}$ for the retailer under the independent channel case:

$$
\left\{\begin{array}{l}
F\left(z^{0}\right)=\frac{z^{0}+(b-1) \Lambda\left(z^{0}\right)}{b z^{0}} \\
p^{0}(z)=\frac{b^{2} c z}{(b-1)^{2}[z-\Lambda(z)]} \\
q^{0}(z)=a p^{-b} z \\
w^{0}=\frac{b}{(b-1)} c
\end{array}\right.
$$

\section{The Wholesale Price Contract Selling to the Pricing-Setting Newsvendor with Social Preferences}

According to [8], social preferences refer to intrinsic concerns for the other party's welfare, reciprocating a history of a positive relationship, and intrinsic desires for a higher relative payoff compared with the other party's when status is salient. Intrinsic preferences regarding relationships and status both take into consideration the welfare of the other parties involved in supply chain interactions. In real-life interactions, their effects are usually mixed with each other and with the economically rational pursuit of payoffs, all effects together influencing observed behavior and actions. As in [8], we define the manufacturer and the retailer's utility functions as follows:

$$
\begin{gathered}
U_{m}=\Pi_{m}+\xi \Pi_{r}, \\
U_{r}=\Pi_{r}+\eta \Pi_{m} .
\end{gathered}
$$

$\xi$ and $\eta$ are called the "other-regarding parameters" of the two players, which reflects the overall concern that each party has for the other party in his/her utility function. In (12), $\xi$ represents the other-regarding parameter of the manufacturer. If $\xi=0$, the manufacturer behaves in a way that is consistent with one not having social preferences and is called traditional. If $\xi<0$, the manufacturer incurs disutility as the retailer's expected profit increases and is called spiteful (or competitive); the larger the absolute value of $\xi$ is, the more spiteful he is. If $\xi>0$, the manufacturer increases utility as the retailer's expected profit increases and is called generous (or altruistic), which implies that the manufacturer cares about improving his or her own expected profit as well as the retailer's. For convenience, we assume that $\frac{1-b}{1+b}<\xi<1$, 
thus $-1<\xi<1$ due to $b>1$. Similarly, $\eta$ in (13) represents the other-regarding parameter of the the retailer. We can also give analogous meaning on $\eta$ and assume that $-1<\eta<1$. It should be noted that the manufacturer (retailer) is a collectivist as $\xi(\eta)$ approaches to 1 , which means the manufacturer (retailer) equally cares about the other's expected profit.

Note that if the manufacturer holds fairness preference that ideal allocation reference points on the fairness preference of inequity aversion are $\frac{\lambda}{1-\lambda}(0<\lambda<1)$. That is to say, the manufacturer regards $\lambda$ as the fairness allocation of the total expected profits for the retailer, leaving $1-\lambda$ as his or her proportion of the total expected profits. At the extremes, if $\lambda=1$, the manufacturer holds 0 as his or her ideal allocation to self $\left(\Pi_{m}=0\right)$, the manufacturer will be generous for any $\Pi_{m}>0$. Similarly, if $\lambda=0$, the manufacturer holds 0 as his or her ideal allocation to the retailer $\left(\Pi_{r}=0\right)$, the manufacturer feels that the retailer obtains more than what he or she deserves and will be spiteful for any $\Pi_{r}>0$. Thus, the utility functions (12) and (13) extend ideal allocations to all or nothing (i.e., $\lambda=0$ or $\lambda=1$ ).

When both the manufacturer and the retailer are with social preferences, it is easy to see the corresponding nine combinations: $\left(\xi_{s}, \xi_{t}, \xi_{g}\right) \times\left(\eta_{s}, \eta_{t}, \eta_{g}\right)$, where $s, t$ and $g$ represent spiteful, traditional and generous preference respectively, and $\frac{1-b}{1+b}<\xi_{s}<0, \xi_{t}=0,0<\xi_{g}<1$; $-1<\eta_{s}<0, \eta_{t}=0,0<\eta_{g}<1$.

Next, we prove Lemma 1.

Lemma 1 Assume that $\frac{1-b}{1+b}<\xi<1$ and $-1<\eta<1$, then $\xi+b-b \xi \eta-1>0$.

Proof To prove that $\xi+b-b \xi \eta-1>0$ is equivalent to prove that $\frac{1-\xi}{1-\xi \eta}<b$.

Since $b>1$ and $\frac{1-b}{1+b}<\xi<1$, it is easy to see that $\max \{1-b,-1\}<\xi<1$. Further, $-1<\eta<1$. Thus, $\xi \eta<1$. Then $0 \leq \frac{1-\xi_{g}}{1-\xi_{g} \eta_{s}} \leq 1-\xi_{g} \leq \frac{1-\xi_{g}}{1-\xi_{g} \eta_{g}}<1<\frac{1-\xi_{s}}{1-\xi_{s} \eta_{g}}<1-\xi_{s}<b$, which implies that $0 \leq \frac{1-\xi_{g}}{1-\xi_{g} \eta_{s}} \leq \frac{1-\xi_{g}}{1-\xi_{g} \eta_{t}} \leq \frac{1-\xi_{g}}{1-\xi_{g} \eta_{g}}<1=\frac{1-\xi_{t}}{1-\xi_{t} \eta_{g}}=\frac{1-\xi_{t}}{1-\xi_{t} \eta_{t}}=\frac{1-\xi_{t}}{1-\xi_{t} \eta_{s}}<\frac{1-\xi_{s}}{1-\xi_{s} \eta_{g}}<$ $\frac{1-\xi_{s}}{1-\xi_{s} \eta_{t}}<b$. Therefore, to complete the proof of Lemma 1, we need further prove that $\frac{1-\xi_{s}}{1-\xi_{s} \eta_{s}}<b$.

Note that $\eta_{s}>-1$, then $-\xi_{s} \eta_{s}>\xi_{s}$, i.e., $1-\xi_{s} \eta_{s}>1+\xi_{s}>0$, thus $\frac{1-\xi_{s}}{1-\xi_{s} \eta_{s}}<\frac{1-\xi_{s}}{1+\xi_{s}}$. On the other hand, due to $\xi_{s}>\frac{1-b}{1+b}$, then $1-\xi_{s}<b+b \xi_{s}$, thus $\frac{1-\xi_{s}}{1+\xi_{s}}<b$. This, in conjunction with $\frac{1-\xi_{s}}{1-\xi_{s} \eta_{s}}<\frac{1-\xi_{s}}{1+\xi_{s}}$, shows that $\frac{1-\xi_{s}}{1-\xi_{s} \eta_{s}}<b$.

When both the manufacturer and the retailer are with social preferences, the retailer's utility function is

$$
\begin{aligned}
U_{r} & =\Pi_{r}+\eta \Pi_{m} \\
& =a p^{-b}\{p[z-\Lambda(z)]-z w\}+\eta a p^{-b} z(w-c) \\
& =a p^{-b}\{p[z-\Lambda(z)]-(1-\eta) z w-\eta z c\},
\end{aligned}
$$

and the manufacturer's utility function is

$$
\begin{aligned}
U_{m} & =\Pi_{m}+\xi \Pi_{r} \\
& =a p^{-b} z(w-c)+\xi a p^{-b}\{p[z-\Lambda(z)]-z w\} \\
& =a p^{-b}\{\xi p[z-\Lambda(z)]+(1-\xi) z w-z c\} .
\end{aligned}
$$

For any fixed $z$, it follows from (14) that

$$
\frac{\partial U_{r}}{\partial p}=a(-b) p^{-(b+1)}\{p[z-\Lambda(z)]-(1-\eta) z w-\eta z c\}+a p^{-b}[z-\Lambda(z)]
$$




$$
=a p^{-(b+1)}\{(1-b)[z-\Lambda(z)] p+b(1-\eta) z w+b \eta z c\} .
$$

Let $\frac{\partial U_{r}}{\partial p}=0$, then

$$
p=\frac{b(1-\eta) z w+b \eta z c}{(b-1)[z-\Lambda(z)]}
$$

Note that $a p^{-(b+1)}>0$ due to $a>0$. Let $R(p)=(1-b)[z-\Lambda(z)] p+b(1-\eta) z w+b \eta z c$, then $R^{\prime}(p)=(1-b)[z-\Lambda(z)]<0$, so $R(p)$ is decreasing in $p$. Therefore, if $p>\frac{b(1-\eta) z w+b \eta z c}{(b-1)[z-\Lambda(z) \mid}$, we have $\frac{\partial U_{r}}{\partial p}<0$; if $p<\frac{b(1-\eta) z w+b \eta z c}{(b-1)[z-\Lambda(z)]}$, we have $\frac{\partial U_{r}}{\partial p}>0$. Thus, $p=\frac{b(1-\eta) z w+b \eta z c}{(b-1)[z-\Lambda(z)]}$ is the unique maximum point of $U_{r}$.

Thus, by (16), we have

$$
w=\frac{(b-1)[z-\Lambda(z)] p-b \eta z c}{(1-\eta) b z} .
$$

By substituting (17) into (15), we have

$$
\begin{aligned}
U_{m} & =a p^{-b}\left\{\xi p[z-\Lambda(z)]+(1-\xi) z \cdot \frac{(b-1)[z-\Lambda(z)] p-b \eta z c}{(1-\eta) b z}-z c\right\} \\
& =a p^{-b}\left\{\frac{(\xi+b-b \eta \xi-1)[z-\Lambda(z)] p+(\eta \xi-1) b z c}{b(1-\eta)}\right\} .
\end{aligned}
$$

For any fixed $z$, by (18), we have

$$
\begin{aligned}
\frac{\partial U_{m}}{\partial p}= & a(-b) p^{-(b+1)} \cdot \frac{(\xi+b-b \eta \xi-1)[z-\Lambda(z)] p+(\eta \xi-1) b z c}{b(1-\eta)} \\
& +a p^{-b} \cdot \frac{(\xi+b-b \eta \xi-1)[z-\Lambda(z)]}{b(1-\eta)} \\
= & a p^{-(b+1)} \cdot \frac{(1-b)(\xi+b-b \eta \xi-1)[z-\Lambda(z)] p+b(1-\eta \xi) b z c}{b(1-\eta)} .
\end{aligned}
$$

Let $\frac{\partial U_{m}}{\partial p}=0$, then

$$
p^{*}(z)=\frac{(1-\eta \xi) b^{2} c z}{(b-1)(\xi+b-b \eta \xi-1)[z-\Lambda(z)]} .
$$

Note that $a p^{-(b+1)}>0$ due to $a>0$. Let

$$
S(p)=\frac{(1-b)(\xi+b-b \eta \xi-1)[z-\Lambda(z)] p+b(1-\eta \xi) b z c}{b(1-\eta)} .
$$

By Lemma 1, we have $S^{\prime}(p)=\frac{(1-b)(\xi+b-b \eta \xi-1)[z-\Lambda(z)]}{b(1-\eta)}<0$, i.e., $S(p)$ is decreasing in $p$. Therefore, if $p>p^{*}(z)$, we have $\frac{\partial U_{m}}{\partial p}<0$; if $p<p^{*}(z)$, we have $\frac{\partial U_{m}}{\partial p}>0$. Thus, $p=p^{*}(z)$ is the unique maximum point of $U_{m}$.

Further, by substituting (20) into (18), we have

$$
\begin{aligned}
U_{m}= & a \cdot \frac{(b-1)^{b}(\xi+b-b \eta \xi-1)^{b}[z-\Lambda(z)]^{b}}{(1-\eta \xi)^{b} b^{2 b} c^{b} z^{b}} \\
& \cdot \frac{(\xi+b-b \eta \xi-1)[z-\Lambda(z)] \cdot \frac{(1-\xi \eta) b^{2} c z}{(b-1)(\xi+b-b \eta \xi-1)[z-\Lambda(z)]}+(\xi \eta-1) b z c}{b(1-\eta)} \\
= & \frac{a(b-1)^{b}(\xi+b-b \eta \xi-1)^{b}[z-\Lambda(z)]^{b}}{(1-\xi \eta)^{b} b^{2 b} c^{b} z^{b}} \cdot \frac{(1-\xi \eta) b c z}{b(b-1)(1-\eta)}
\end{aligned}
$$




$$
=\frac{a(b-1)^{b-1}(\xi+b-b \eta \xi-1)^{b}}{(1-\eta)(1-\xi \eta)^{b-1} b^{2 b} c^{b-1}} \cdot \frac{[z-\Lambda(z)]^{b}}{z^{b-1}} .
$$

Thus,

$$
\begin{aligned}
\frac{\partial U_{m}}{\partial z} & =\frac{a(b-1)^{b-1}(\xi+b-b \eta \xi-1)^{b}}{(1-\eta)(1-\xi \eta)^{b-1} b^{2 b} c^{b-1}} \cdot \frac{b[z-\Lambda(z)]^{b-1}[1-F(z)] z^{b-1}-[z-\Lambda(z)]^{b}(b-1) z^{b-2}}{z^{2(b-1)}} \\
& =\frac{a(b-1)^{b-1}(\xi+b-b \eta \xi-1)^{b}}{(1-\eta)(1-\xi \eta)^{b-1} b^{2 b} c^{b-1}} \cdot \frac{[z-\Lambda(z)]^{b-1}}{z^{b}}\{z+(b-1) \Lambda(z)-b z F(z)\} .
\end{aligned}
$$

Note that $\frac{a(b-1)^{b-1}(\xi+b-b \eta \xi-1)^{b}}{(1-\eta)(1-\xi \eta)^{b-1} b^{2 b} b^{b-1}} \cdot \frac{[z-\Lambda(z)]^{b-1}}{z^{b}}>0$. Let $H(z)=z-b z F(z)+(b-1) \Lambda(z)$, and implement the same analysis as (4), we have

$$
F\left(z^{*}\right)=\frac{z^{*}+(b-1) \Lambda\left(z^{*}\right)}{b z^{*}} .
$$

On the other hand, by substituting (20) into (17), we have

$$
w^{*}=\frac{(b-1)[z-\Lambda(z)] \cdot \frac{(1-\eta \xi) b^{2} c z}{(b-1)(\xi+b-b \eta \xi-1)[z-\Lambda(z)]}-b \eta z c}{(1-\eta) b z}=\frac{b c\left(1-\xi \eta-\eta+\xi \eta^{2}\right)+\eta c(1-\xi)}{(1-\eta)(\xi+b-b \eta \xi-1)} .
$$

In summary, we have the following optimal wholesale price $w^{*}$ for the manufacturer, the optimal retail price $p^{*}$ and order quantity $q^{*}$ for the retailer when both the manufacturer and the retailer are with social preferences:

$$
\left\{\begin{array}{l}
F\left(z^{*}\right)=\frac{z^{*}+(b-1) \Lambda\left(z^{*}\right)}{b z^{*}} \\
p^{*}(z)=\frac{(1-\xi \eta) b^{2} c z}{(b-1)(\xi+b-b \eta \xi-1)[z-\Lambda(z)]} \\
q^{*}(z)=a p^{-b} z \\
w^{*}=\frac{b c\left(1-\xi \eta-\eta+\xi \eta^{2}\right)+\eta c(1-\xi)}{(1-\eta)(\xi+b-b \eta \xi-1)}
\end{array}\right.
$$

\subsection{Only the Retailer Is with Social Preferences}

Let $\xi=0$ in (23), then, we have the following optimal wholesale price $w^{*}$ for the manufacturer, the optimal retail price $p^{*}$ and order quantity $q^{*}$ for the retailer when only the retailer is with social preferences:

$$
\left\{\begin{array}{l}
F\left(z^{*}\right)=\frac{z^{*}+(b-1) \Lambda\left(z^{*}\right)}{b z^{*}} \\
p^{*}(z)=\frac{b^{2} c z}{(b-1)^{2}[z-\Lambda(z)]} \\
q^{*}(z)=a p^{-b} z \\
w^{*}=\frac{\eta+b-b \eta}{(b-1)(1-\eta)} c .
\end{array}\right.
$$

Compared to the independent channel case, it is easy to see that that $F\left(z^{*}\right)=F\left(z^{0}\right), p^{*}(z)=$ $p^{0}(z), q^{*}(z)=q^{0}(z)$. Therefore, the only influential decision variable is the manufacturer's wholesale price when only the retailer is with social preferences.

Next, we study how the retailer's other-regarding parameter $\eta$ affects the manufacturer's wholesale price. Note that $w^{*}-w^{0}=\frac{\eta+b(1-\eta)}{(b-1)(1-\eta)} c-\frac{b}{b-1} c=\frac{\eta c}{(b-1)(1-\eta)}$, thus:

1) If $\eta=0$, we have $w^{*}=w^{0}=\frac{b}{b-1} c$, which implies that the manufacturer's optimal wholesale price is the same as that in the traditional one when the retailer is traditional. 
2) If $0<\eta<1$, we have $w^{*}-w^{0}>0$, i.e., $w^{*}>w^{0}$, which implies that the manufacturer's optimal wholesale price is greater than that in the traditional one when the retailer is generous. We explain this phenomenon as follows: The generous retailer orders more than a traditional retailer when the manufacturer sets the same wholesale price. With this issue, the manufacturer facing a generous retailer will set a wholesale price larger than that facing a traditional retailer. The decreasing trend of the retailer's order quantity on the wholesale price is moderated by the retailer's generosity. It is then optimal for the manufacturer to increase the wholesale price to the point at which the generous retailer will order exactly the traditional quantity. So, when only the retailer is with social preferences, the manufacturer's optimal wholesale price is affected, but the optimal retail price and order quantity for the retailer are the same as those in traditional one. That is to say, the retailer's other-regarding parameter affects the profit allocation between the manufacturer and the retailer.

3) If $-1<\eta<0$, we have $w^{*}-w^{0}=<0$, i.e., $w^{*}<w^{0}$, which implies that the manufacturer's optimal wholesale price is smaller than that in the traditional one when the retailer is spiteful. Under this case, we can implement the analogous analysis as $0<\eta<1$.

In the following, we study the influence of the retailer's other-regarding parameter on the profits and utilities of the supply chain member and the whole system.

Theorem 1 When only the retailer is with social preferences, the retailer's profit decreases as $\eta$ increases, the manufacturer's profit increases as $\eta$ increases, and the system's profit is independent of $\eta$.

Proof By substituting (24) into (8), we have

$$
\begin{aligned}
\Pi_{r}^{*} & =a p^{-b}\{p[z-\Lambda(z)]-z w\} \\
& =a \frac{(b-1)^{2 b}[z-\Lambda(z)]^{b}}{b^{2 b} c^{b} z^{b}}\left\{\frac{b^{2} c z}{(b-1)^{2}[z-\Lambda(z)]} \cdot[z-\Lambda(z)]-\frac{\eta+b-b \eta}{(b-1)(1-\eta)} c z\right\} \\
& =\frac{a(b-1)^{2 b-2}[z-\Lambda(z)]^{b}}{b^{2 b} c^{b-1} z^{b-1}} \cdot \frac{\eta-2 b \eta+b}{1-\eta} .
\end{aligned}
$$

then, $\frac{\mathrm{d} \Pi_{r}}{\mathrm{~d} \eta}=\frac{a(b-1)^{2 b-2}[z-\Lambda(z)]^{b}}{b^{2 b} c^{b-1} z^{b-1}} \cdot \frac{1-b}{(1-\eta)^{2}}<0$. Thus, the retailer's profit decreases as $\eta$ increases.

By substituting (24) into (9), we have

$$
\begin{aligned}
\Pi_{m}^{*}=w q-c q & =a p^{-b} z(w-c) \\
& =a \frac{(b-1)^{2 b}[z-\Lambda(z)]^{b} z}{b^{2 b} c^{b} z^{b}}\left[\frac{\eta+b-b \eta}{(b-1)(1-\eta)} c-c\right] \\
& =\frac{a(b-1)^{2 b-1}[z-\Lambda(z)]^{b}}{b^{2 b} c^{b-1} z^{b-1}(1-\eta)} .
\end{aligned}
$$

then, $\frac{\mathrm{d} \Pi_{m}}{\mathrm{~d} \eta}=\frac{a(b-1)^{2 b-1}[z-\Lambda(z)]^{b}}{b^{2 b} c^{b-1} z^{b-1}} \cdot \frac{1}{(1-\eta)^{2}}>0$. Thus, the manufacturer's profit increases as $\eta$ increases.

Moreover, the supply chain system's profit is

$$
\Pi^{*}=\Pi_{r}^{*}+\Pi_{m}^{*}=a p^{-b}\{p[z-\Lambda(z)]-z w\}+a p^{-b} z(w-c)=a p^{-b}\{p[z-\Lambda(z)]-c z\} .
$$

Note that the above forma is dependent on $p$ and $z$, but independent of $w$. Furthermore, by (24), it is easy to see that $p$ and $z$ are independent of $\eta$. Thus, the system's profit of the supply 
chain is independent of $\eta$. That is to say, the retailer's other-regarding parameter has no effects on the supply chain system's profit.

Theorem 2 When only the retailer is with social preferences, the retailer's utility is independent of $\eta$, the supply chain system's utility increases as $\eta$ increases, thus the manufacturer's utility increases as $\eta$ increases.

Proof By (14), the retailer's utility function is

$$
U_{r}=a p^{-b}\{p[z-\Lambda(z)]-z w+\eta z(w-c)\} .
$$

By substituting (24) into the above forma, we have

$$
\begin{aligned}
U_{r}^{*}= & a \frac{(b-1)^{2 b}[z-\Lambda(z)]^{b}}{b^{2 b} c^{b} z^{b}}\left\{\frac{b^{2} c z}{(b-1)^{2}[z-\Lambda(z)]} \cdot[z-\Lambda(z)]-\frac{(\eta+b-b \eta) c z}{(b-1)(1-\eta)}\right. \\
& \left.+\eta z\left[\frac{\eta+b-b \eta}{(b-1)(1-\eta)} c-c\right]\right\} \\
= & \frac{a(b-1)^{2 b-2}[z-\Lambda(z)]^{b}}{b^{2 b-1} c^{b-1} z^{b-1}} .
\end{aligned}
$$

This implies that the retailer's utility function is independent of $\eta$. That is to say, the retailer's utility remains unchanged when only the retailer is with social preferences.

The supply chain system's utility is

$$
U=U_{r}+U_{m}=a p^{-b}\{p[z-\Lambda(z)]-z w+\eta z(w-c)\}+a p^{-b} z(w-c) .
$$

By substituting (24) into the above forma, we have

$$
U^{*}=\frac{a(b-1)^{2 b-2}[z-\Lambda(z)]^{b}}{b^{2 b-1} c^{b-1} z^{b-1}}\left[1+\frac{b-1}{b(1-\eta)}\right],
$$

then,

$$
\frac{\mathrm{d} U^{*}}{\mathrm{~d} \eta}=\frac{a(b-1)^{2 b-1}[z-\Lambda(z)]^{b}}{b^{2 b} c^{b-1} z^{b-1}} \cdot \frac{1}{(1-\eta)^{2}}>0 .
$$

Thus, the supply chain system's utility increases as $\eta$ increases. This, in conjunction with that the retailer's utility remains unchanged, shows that the manufacturer's utility increases as $\eta$ increases.

\subsection{Only the Manufacturer Is with Social Preferences}

Let $\eta=0$ in (23), then, we have the following optimal wholesale price $w^{*}$ for the manufacturer, the optimal retail price $p^{*}$ and order quantity $q^{*}$ for the retailer when only the manufacturer is with social preferences:

$$
\left\{\begin{array}{l}
F\left(z^{*}\right)=\frac{z^{*}+(b-1) \Lambda\left(z^{*}\right)}{b z^{*}} \\
p^{*}(z)=\frac{b^{2} c z}{(b-1)(b-1+\xi)[z-\Lambda(z)]} \\
q^{*}(z)=a p^{-b} z \\
w^{*}=\frac{b c}{b-1+\xi}
\end{array}\right.
$$

Obviously, compared with the case that only the retailer is with social preferences, the manufacturer's other-regarding parameter $\xi$ has effects on the optimal wholesale price for the manufacturer, the optimal retail price and order quantity for the retailer. 
Note that $F\left(z^{*}\right)=F\left(z^{0}\right)$, thus $z^{*}=z^{0}$. Furthermore, $p^{*}(z)-p^{0}(z)=\frac{b^{2} c z}{(b-1)(b-1+\xi)[z-\Lambda(z)]}-$ $\frac{b^{2} c z}{(b-1)^{2}[z-\Lambda(z)]}=\frac{-\xi b^{2} c z}{(b-1)^{2}(b-1+\xi)[z-\Lambda(z)]} ; \frac{q^{*}(z)}{q^{0}(z)}=\left(\frac{p^{*}(z)}{p^{0}(z)}\right)^{-b}=\left(\frac{b-1}{b-1+\xi}\right)^{-b} ; w^{*}-w^{0}=\frac{b c}{b-1+\xi}-\frac{b c}{b-1}=$ $-\frac{\xi b c}{(b-1)(b-1+\xi)}$.

Next, we study how the degree of the manufacturer's other-regarding parameter $\xi$ affects the manufacturer's wholesale price, the retailer's optimal order quantity and retail price. We consider the following three cases:

1) If $\xi=0$, i.e., the manufacturer is traditional, we have $w^{*}=w^{0} ; p^{*}=p^{0} ; q^{*}=q^{0}$.

2) If $0<\xi<1$, i.e., the manufacturer is generous, we have $w^{*}-w^{0}<0$, i.e., $w^{*}<w^{0}$, which implies that the manufacturer sets the wholesale price smaller than the traditional one; $p^{*}-p^{0}<0$, i.e., $p^{*}<p^{0}$, which implies that the retailer's optimal retail price is smaller than the traditional one; $\frac{q^{*}(z)}{q^{0}(z)}>1$, i.e., $q^{*}>q^{0}$, which implies that the retailer's optimal order quantity is greater than the traditional one.

3) If $\frac{1-b}{1+b}<\xi<0$, i.e., the manufacturer is spiteful, we have $w^{*}>w^{0} ; p^{*}>p^{0} ; q^{*}<q^{0}$. Under this case, we obtain the contrary results in 2).

In the following, we study the influence of the manufacturer's other-regarding parameter on the profits and utilities of the supply chain member and the whole system.

Theorem 3 When only the manufacturer is with social preferences, the retailer and the whole system's profits increase as $\xi$ increases; the manufacturer's profit first increases, then decreases as $\xi$ increases, and the manufacturer's profit achieves the maximization as $\xi=0$.

Proof By substituting (25) into (8) and (9) respectively, we have

$$
\begin{aligned}
\Pi_{r}^{*} & =\frac{a(b-1)^{b-1}(b-1+\xi)^{b-1}[z-\Lambda(z)]^{b}}{b^{2 b-1} c^{b-1} z^{b-1}}, \\
\Pi_{m}^{*} & =\frac{a(b-1)^{b}(b-1+\xi)^{b-1}(1-\xi)[z-\Lambda(z)]^{b}}{b^{2 b} c^{b-1} z^{b-1}} .
\end{aligned}
$$

Then, $\frac{\mathrm{d} \Pi_{r^{*}}}{\mathrm{~d} \xi}=\frac{a(b-1)^{b}(b-1+\xi)^{b-2}[z-\Lambda(z)]^{b}}{b^{2 b-1} c^{b-1} z^{b-1}}>0$, which implies that the retailer's profit increases as $\xi$ increases. On the other hand, $\frac{\mathrm{d} \Pi_{m^{*}}}{\mathrm{~d} \xi}=-\frac{a(b-1)^{b}(b-1+\xi)^{b-2} \xi[z-\Lambda(z)]^{b}}{b^{2 b-1} c^{b-1} z^{b-1}}$. Thus, if $\frac{1-b}{1+b}<\xi<0$, we have $\frac{\mathrm{d} \Pi_{m^{*}}}{\mathrm{~d} \xi}>0$, which implies that the manufacturer's profit increases as $\eta$ increases; if $0<\xi<1$, we have $\frac{\mathrm{d} \Pi_{m^{*}}}{\mathrm{~d} \xi}<0$, which implies that the manufacturer's profit decreases as $\eta$ increases. Therefore, the manufacturer's profit achieves the maximization as $\xi=0$.

Moreover, the system's profit is

$$
\begin{aligned}
\Pi^{*} & =\Pi_{r}^{*}+\Pi_{m}^{*} \\
& =\frac{a(b-1)^{b-1}(b-1+\xi)^{b-1}[z-\Lambda(z)]^{b}}{b^{2 b-1} c^{b-1} z^{b-1}}+\frac{a(b-1)^{b}(b-1+\xi)^{b-1}(1-\xi)[z-\Lambda(z)]^{b}}{b^{2 b} c^{b-1} z^{b-1}} \\
& =\frac{a(b-1)^{b-1}[z-\Lambda(z)]^{b}}{b^{2 b-1} c^{b-1} z^{b-1}}(b-1+\xi)^{b-1}\left[1+\frac{(b-1)(1-\xi)}{b}\right] .
\end{aligned}
$$

Note that $\frac{a(b-1)^{b-1}[z-\Lambda(z)]^{b}}{b^{2 b-1} c^{b-1} z^{b-1}}>0$. Let

$$
u(\xi)=(b-1+\xi)^{b-1}\left[1+\frac{(b-1)(1-\xi)}{b}\right]
$$

then

$$
u^{\prime}(\xi)=(b-1)(b-1+\xi)^{b-2}(1-\xi)>0
$$


which implies that $u(\xi)$ is increasing in $\xi$. Thus, $\Pi^{*}$ is increasing in $\xi$, which implies that the system's profit increases as $\xi$ increases.

Theorem 4 When only the manufacturer is with social preferences, the manufacturer's, the retailer's and the system's utilities of the supply chain all increase as $\xi$ increases.

Proof By (15), the manufacturer's utility function is

$$
U_{m}=a p^{-b}\{\xi p[z-\Lambda(z)]+(1-\xi) z w-z c\} .
$$

By substituting (25) into the above forma, we have

$$
U_{m}^{*}=\frac{a(b-1)^{b-1}(b-1+\xi)^{b}[z-\Lambda(z)]^{b}}{b^{2 b} c^{b-1} z^{b-1}} .
$$

Then,

$$
\frac{\mathrm{d} U_{m}^{*}}{\mathrm{~d} \xi}=\frac{a(b-1)^{b-1}[z-\Lambda(z)]^{b}}{b^{2 b-1} c^{b-1} z^{b-1}}(b-1+\xi)^{b-1}>0,
$$

which implies that the manufacturer's utility increases as $\xi$ increases. Similarly, the retailer's utility function is

$$
U_{r}=\Pi_{r}=a p^{-b}\{p[z-\Lambda(z)]-z w\}=a p^{-b}\{p[z-\Lambda(z)]-z w\} .
$$

By substituting (25) into the above forma, we have

$$
U_{r}^{*}=\frac{a(b-1)^{b-1}(b-1+\xi)^{b-1}[z-\Lambda(z)]^{b}}{b^{2 b-1} c^{b-1} z^{b-1}} .
$$

Then, $\frac{\mathrm{d} U_{r}^{*}}{\mathrm{~d} \xi}=\frac{a(b-1)^{b}[z-\Lambda(z)]^{b}}{b^{2 b-1} c^{b-1} z^{b-1}}(b-1+\xi)^{b-2}>0$, which implies that the retailer's utility increases as $\xi$ increases.

Moreover, the system's utility function is

$$
\begin{aligned}
U^{*} & =U_{r}^{*}+U_{m}^{*} \\
& =\frac{a(b-1)^{b-1}(b-1+\xi)^{b-1}[z-\Lambda(z)]^{b}}{b^{2 b-1} c^{b-1} z^{b-1}}+\frac{a(b-1)^{b-1}(b-1+\xi)^{b}[z-\Lambda(z)]^{b}}{b^{2 b} c^{b-1} z^{b-1}} \\
& =\frac{a(b-1)^{b-1}[z-\Lambda(z)]^{b}}{b^{2 b-1} c^{b-1} z^{b-1}} \cdot(b-1+\xi)^{b-1}\left(1+\frac{b-1+\xi}{b}\right) .
\end{aligned}
$$

Note that $\frac{a(b-1)^{b-1}[z-\Lambda(z)]^{b}}{b^{2 b-1} c^{b-1} z^{b-1}}>0$. Let

$$
v(\xi)=(b-1+\xi)^{b-1}\left(1+\frac{b-1+\xi}{b}\right),
$$

then

$$
v^{\prime}(\xi)=(b-1+\xi)^{b-2}(2 b-2+\xi)>0,
$$

which implies that $v(\xi)$ is increasing in $\xi$. Thus, $U^{*}$ is increasing in $\xi$, which implies that the system's utility increases as $\xi$ increases.

By Theorems 1 and 3, the retailer's other-regarding parameter $\eta$ does not affect the supply chain system's profit; but the manufacturer's other-regarding parameter $\xi$ has significant effects on the supply chain system's profit. The more generous the manufacturer is, the more the system's profit is, vice versa. The essential reason is that the manufacturer is a leader in this Stackelberg game. We will explore further this point in Subsection 3.3. 


\subsection{Both the Manufacturer and the Retailer Are with Social Preferences}

When both the manufacturer and the retailer are with social preferences, let $p^{i j}, q^{i j}$ and $\Pi^{i j}(i, j=s, g, t)$ denote the retailer's optimal retail price, order quantity and the system's profit given that the manufacturer's and the retailer's other-regarding parameter preferences are $i$ and $j$, respectively.

Theorem 5 When both the manufacturer and the retailer are with social preferences, the optimal retail prices under nine combinations satisfy the following relations: $p^{g s}<p^{g t}<p^{g g}<$ $p^{t g}=p^{t t}=p^{t s}<p^{s g}<p^{s t}<p^{s s}$; the optimal order quantities under nine combinations satisfy the following relations: $q^{g s}>q^{g t}>q^{g g}>q^{t g}=q^{t t}=q^{t s}>q^{s g}>q^{s t}>q^{s s}$.

Proof First, we consider the relationships between the optimal retail prices under nine combinations. By (23), we have $p=\frac{(1-\xi \eta) b^{2} c z}{(b-1)(\xi+b-b \xi \eta-1)[z-\Lambda(z)]}$. Let $p_{1}=\frac{1-\xi \eta}{\xi+b-b \xi \eta-1}$, then $p=$ $p_{1} \cdot \frac{b^{2} c z}{(b-1)[z-\Lambda(z)]}$.

1) Note that $p_{1}^{s s}=\frac{1-\xi_{s} \eta_{s}}{\xi_{s}+b-b \xi_{s} \eta_{s}-1}$ and $p_{1}^{s t}=\frac{1}{\xi_{s}+b-1}$, then

$p_{1}^{s s}-p_{1}^{s t}=\frac{\left(1-\xi_{s} \eta_{s}\right)\left(\xi_{s}+b-1\right)-\left(\xi_{s}+b-b \xi_{s} \eta_{s}-1\right)}{\left(\xi_{s}+b-b \xi_{s} \eta_{s}-1\right)\left(\xi_{s}+b-1\right)}=\frac{\xi_{s} \eta_{s}\left(1-\xi_{s}\right)}{\left(\xi_{s}+b-b \xi_{s} \eta_{s}-1\right)\left(\xi_{s}+b-1\right)}>0$, i.e., $p_{1}^{s s}>p_{1}^{s t}$. Thus, $p^{s s}>p^{s t}$.

2) Note that $p_{1}^{s t}=\frac{1}{\xi_{s}+b-1}$ and $p_{1}^{s g}=\frac{1-\xi_{s} \eta_{g}}{\xi_{s}+b-b \xi_{s} \eta_{g}-1}$, then

$p_{1}^{s t}-p_{1}^{s g}=\frac{\left(\xi_{s}+b-b \xi_{s} \eta_{g}-1\right)-\left(1-\xi_{s} \eta_{g}\right)\left(\xi_{s}+b-1\right)}{\left(\xi_{s}+b-b \xi_{s} \eta_{g}-1\right)\left(\xi_{s}+b-1\right)}=\frac{\xi_{s} \eta_{g}\left(\xi_{s}-1\right)}{\left(\xi_{s}+b-b \xi_{s} \eta_{s}-1\right)\left(\xi_{s}+b-1\right)}>0$, i.e., $p_{1}^{s t}>p_{1}^{s g}$. Thus, $p^{s t}>p^{s g}$.

3) Note that $p_{1}^{s g}=\frac{1-\xi_{s} \eta_{g}}{\xi_{s}+b-b \xi_{s} \eta_{g}-1}$ and $p_{1}^{t t}=\frac{1}{b-1}$, then

$$
p_{1}^{s g}-p_{1}^{t t}=\frac{\left(1-\xi_{s} \eta_{g}\right)(b-1)-\left(\xi_{s}+b-b \xi_{s} \eta_{g}-1\right)}{(b-1)\left(\xi_{s}+b-b \xi_{s} \eta_{g}-1\right)}=\frac{\xi_{s}\left(\eta_{g}-1\right)}{(b-1)\left(\xi_{s}+b-b \xi_{s} \eta_{g}-1\right)}>0,
$$

i.e., $p_{1}^{s g}>p_{1}^{t t}$. Thus, $p^{s g}>p^{t t}$.

4) Note that $p_{1}^{t t}=\frac{1}{b-1}$ and $p_{1}^{g g}=\frac{1-\xi_{g} \eta_{g}}{\xi_{g}+b-b \xi_{g} \eta_{g}-1}$, then

$$
p_{1}^{t t}-p_{1}^{g g}=\frac{\left(\xi_{g}+b-b \xi_{g} \eta_{g}-1\right)-(b-1)\left(1-\xi_{g} \eta_{g}\right)}{(b-1)\left(\xi_{g}+b-b \xi_{g} \eta_{g}-1\right)}=\frac{\xi_{g}\left(1-\eta_{g}\right)}{(b-1)\left(\xi_{g}+b-b \xi_{g} \eta_{g}-1\right)}>0,
$$

i.e., $p_{1}^{t t}>p_{1}^{g g}$. Thus, $p^{t t}>p^{g g}$.

5) Note that $p_{1}^{g g}=\frac{1-\xi_{g} \eta_{g}}{\xi_{g}+b-b \xi_{g} \eta_{g}-1}$ and $p_{1}^{g t}=\frac{1}{\xi_{g}+b-1}$, then

$p_{1}^{g g}-p_{1}^{g t}=\frac{\left(1-\xi_{g} \eta_{g}\right)\left(\xi_{g}+b-1\right)-\left(\xi_{g}+b-b \xi_{g} \eta_{g}-1\right)}{\left(\xi_{g}+b-b \xi_{g} \eta_{g}-1\right)\left(\xi_{g}+b-1\right)}=\frac{\xi_{g} \eta_{g}\left(1-\xi_{g}\right)}{\left(\xi_{g}+b-b \xi_{g} \eta_{g}-1\right)\left(\xi_{g}+b-1\right)}>0$, i.e., $p_{1}^{g g}>p_{1}^{g t}$. Thus, $p^{g g}>p^{g t}$.

6) Note that $p_{1}^{g t}=\frac{1}{\xi_{g}+b-1}$ and $p_{1}^{g s}=\frac{1-\xi_{g} \eta_{s}}{\xi_{g}+b-b \xi_{g} \eta_{s}-1}$, then

$p_{1}^{g t}-p_{1}^{g s}=\frac{\left(\xi_{g}+b-b \xi_{g} \eta_{s}-1\right)-\left(1-\xi_{g} \eta_{s}\right)\left(\xi_{g}+b-1\right)}{\left(\xi_{g}+b-1\right)\left(\xi_{g}+b-b \xi_{g} \eta_{s}-1\right)}=\frac{\xi_{g} \eta_{s}\left(\xi_{g}-1\right)}{\left(\xi_{g}+b-1\right)\left(\xi_{g}+b-b \xi_{g} \eta_{s}-1\right)}>0$, i.e., $p_{1}^{g t}>p_{1}^{g s}$. Thus, $p^{g t}>p^{g s}$.

Moreover, by Subsection 3.1, we have $p^{t g}=p^{t t}=p^{t s}$ when only the retailer is with social preferences. Thus, $p^{g s}<p^{g t}<p^{g g}<p^{t g}=p^{t t}=p^{t s}<p^{s g}<p^{s t}<p^{s s}$. 
Next, we consider the relationships between the optimal order quantities under nine combinations. By (23), $q=a p^{-b} z$, then $\frac{q^{g s}}{q^{g t}}=\frac{a\left(p^{g s}\right)^{-b} z}{a\left(p^{g t}\right)^{-b} z}=\left(\frac{p^{g t}}{p^{g s}}\right)^{b}$. Moreover, $p^{g t}>p^{g s}$, i.e., $\frac{p^{g t}}{p^{g s}}>1$. It is easy to see that $\frac{q^{g s}}{q^{g t}}>1$ by the property of power function. Thus, $q^{g s}>q^{g t}$. Implementing the analogous analysis, we have $q^{g t}>q^{g g}>q^{t g}=q^{t t}=q^{t s}>q^{s g}>q^{s t}>q^{s s}$. The proof is complete.

Theorem 6 When both the manufacturer and the retailer are with social preferences, the optimal system's profits under nine combinations satisfy the following relations: $\Pi^{g s}>\Pi^{g t}>$ $\Pi^{g g}>\Pi^{t g}=\Pi^{t t}=\Pi^{t s}>\Pi^{s g}>\Pi^{s t}>\Pi^{s s}$.

Proof By (8) and (9), the system's profit under the independent channel case is

$$
\Pi=\Pi_{r}+\Pi_{m}=a p^{-b}\{p[z-\Lambda(z)]-z c\}=a p^{-b+1}[z-\Lambda(z)]-a p^{-b} z c .
$$

Let

$$
r(p):=a p^{-b+1}[z-\Lambda(z)]-a p^{-b} z c,
$$

then

$$
r^{\prime}(p)=a(-b+1) p^{-b}[z-\Lambda(z)]+a b p^{-b-1} z c=a p^{-b}\left[(b-1) \Lambda(z)+z-b z+\frac{b z c}{p}\right] .
$$

By (23), $(b-1) \Lambda(z)+z=b z F(z)$. Thus, $r^{\prime}(p)=a p^{-b} b z\left[F(z)-1+\frac{c}{p}\right]$.

In the following, we prove that $F(z)-1+\frac{c}{p}<0$. By $(23), \frac{c}{p}=\frac{(b-1)(\xi+b-b \xi \eta-1)[z-\Lambda(z)]}{(1-\xi \eta) b^{2} z}$, $1-F(z)=\frac{(b-1)[z-\Lambda(z)]}{b z}$. Thus,

$\frac{c}{p}-1+F(z)=\frac{(b-1)[z-\Lambda(z)]}{b z} \cdot\left(\frac{\xi+b-b \xi \eta-1}{(1-\xi \eta) b}-1\right)=\frac{(b-1)[z-\Lambda(z)]}{b z} \cdot \frac{\xi-1}{(1-\xi \eta) b}<0$.

This, in conjunction with $r^{\prime}(p)=a p^{-b} b z\left[F(z)-1+\frac{c}{p}\right]$, shows that $r(p)$ is decreasing in $p$. Moreover, by Theorem 5, $p^{g s}<p^{g t}<p^{g g}<p^{t g}=p^{t t}=p^{t s}<p^{s g}<p^{s t}<p^{s s}$. Thus, $\Pi^{g s}>\Pi^{g t}>\Pi^{g g}>\Pi^{t g}=\Pi^{t t}=\Pi^{t s}>\Pi^{s g}>\Pi^{s t}>\Pi^{s s}$.

By Theorem 6, the supply chain suffers the most when the manufacturer and the retailer are spiteful. However, the supply chain performance is not the best when both players are generous, but instead when the manufacturer is generous and the retailer is spiteful. Therefore, to improve the entire supply chain (i.e., with larger profit), it is necessary that the manufacturer is generous. Otherwise, the system's profit diminishes whether the retailer is generous or not. The essential reason is that the manufacturer is a leader in this Stackelberg game. Thus, we obtain the following important insights: The manufacturer, as the leader, should be in charge of the supply chain. The retailer, as the follower, is always in passive status no matter his or her type is. That is, the retailer should do his or her best work without considering the whole supply chain. Therefore, the manufacturer behaves like a government with considering the total social utility of the nation; the retailer behaves like persons in a nation: Believing that doing his or her best will benefit the total social utility of the nation, as explained by Adam Smith's theory of the invisible hand in the market.

In addition, the relationships are complex on the manufacturer's optimal wholesale price, the supply chain member and system's profits and utilities under nine combinations. We will illustrate them in the next section. 
At last, by (23), as $\xi$ approaches to 1, the retailer's optimal order quantity and retailer price are the same as (6) under the integrated channel case, and the manufacturer's optimal wholesale price equals to marginal product cost $c$. The extremely generous manufacturer coordinates the supply chain.

\section{Numerical Examples}

For price-dependent stochastic demand function $D(p, \varepsilon)=y(p) \varepsilon$, it is assumed that $\varepsilon$ follows the uniform distribution on the interval $[0, h]$, then $F(x)=\frac{x}{h}(0 \leq x \leq h), f(x)=\frac{1}{h}, \Lambda(z)=\frac{z^{2}}{2 h}$. By substituting them into (23), we have

$$
\left\{\begin{aligned}
z^{*} & =\frac{2 h}{b+1} \\
w^{*} & =\frac{b c\left(1-\xi \eta-\eta+\xi \eta^{2}\right)+\eta c(1-\xi)}{(1-\eta)(\xi+b-b \eta \xi-1)} \\
p^{*} & =\frac{(b+1)(1-\xi \eta) b c}{(b-1)(\xi+b-b \eta \xi-1)} \\
q^{*} & =\frac{2 a h(b-1)^{b}(\xi+b-b \eta \xi-1)^{b}}{b^{b} c^{b}(b+1)^{b+1}(1-\xi \eta)^{b}}
\end{aligned}\right.
$$

Further, let $h=2$, and assume that $y(p)=1000 p^{-2}$, i.e., $a=1000$ and $b=2$. Moreover, the manufacturer's marginal cost $c$ equals to 2 . By (11), we can obtain that the manufacturer's optimal wholesale price is $w^{0}=4$, the retailer's optimal order quantity $q^{0}=9.259$ and optimal retail price $p^{0}=12$, and the system's optimal profit is 55.556 under the independent channel case.

Let $\xi=0$ in (26), we study that the retailer's other-regarding parameter $\eta$ has effects on the supply chain system. By (26), we can obtain that the manufacturer's optimal wholesale price $w^{*}$, the retailer's optimal order quantity $q^{*}$ and optimal retail price $p^{*}$; Furthermore, we can obtain the retailer's profit $\Pi_{r}^{*}$ and utility $U_{r}^{*}$, the manufacturer's profit $\Pi_{m}^{*}$ and utility $U_{m}^{*}$, the supply chain system's profit $\Pi^{*}$ and utility $U^{*}$. These results are shown in Table 1 .

Table 1 The supply chain system when only the retailer is with social preferences

\begin{tabular}{ccccccccc}
\hline$\eta$ & $w^{*}$ & $q^{*}$ & $p^{*}$ & $\Pi_{r}^{*}$ & $U_{r}^{*}$ & $\Pi_{m}^{*}\left(=U_{m}^{*}\right)$ & $\Pi^{*}$ & $U^{*}$ \\
\hline-0.8 & 3.111 & 9.259 & 12.000 & 45.268 & 37.037 & 10.288 & 55.556 & 47.325 \\
-0.6 & 3.25 & 9.259 & 12.000 & 43.982 & 37.037 & 11.574 & 55.556 & 48.611 \\
-0.4 & 3.429 & 9.259 & 12.000 & 42.328 & 37.037 & 13.228 & 55.556 & 50.265 \\
-0.2 & 3.667 & 9.259 & 12.000 & 40.124 & 37.037 & 15.432 & 55.556 & 52.469 \\
0 & 4.000 & 9.259 & 12.000 & 37.037 & 37.037 & 18.519 & 55.556 & 55.556 \\
0.2 & 4.500 & 9.259 & 12.000 & 32.407 & 37.037 & 23.148 & 55.556 & 60.185 \\
0.4 & 5.330 & 9.259 & 12.000 & 24.691 & 37.037 & 30.864 & 55.556 & 67.901 \\
0.6 & 7.000 & 9.259 & 12.000 & 9.259 & 37.037 & 46.296 & 55.556 & 83.333 \\
0.8 & 12.000 & 9.259 & 12.000 & -37.037 & 37.037 & 92.593 & 55.556 & 129.630 \\
\hline
\end{tabular}

Similarly, let $\eta=0$ in (26), we study that the manufacturer's other-regarding parameter $\xi$ has effects on the supply chain system. Note that we assume that $\frac{1-b}{1+b}<\xi<1$. Thus, 
$\frac{-1}{3}<\xi<1$ as $b=2$. But, on the other hand, we can conclude that (23) holds as $-1<\xi<1$ by letting $\eta=0$ in (14) and checking the deductions. Therefore, under this case, we obtain Table 2 .

Table 2 The supply chain system when only the manufacturer is with social preferences

\begin{tabular}{ccccccccc}
\hline$\xi$ & $w^{*}$ & $q^{*}$ & $p^{*}$ & $\Pi_{r}^{*}=\left(U_{r}^{*}\right)$ & $\Pi_{m}^{*}$ & $U_{m}^{*}$ & $\Pi^{*}$ & $U^{*}$ \\
\hline-0.8 & 20.000 & 0.370 & 60.000 & 7.407 & 6.667 & 0.741 & 14.074 & 8.148 \\
-0.6 & 10.000 & 1.481 & 30.000 & 14.815 & 11.852 & 2.963 & 26.667 & 17.778 \\
-0.4 & 6.667 & 3.333 & 20.000 & 22.222 & 15.556 & 6.667 & 37.778 & 28.889 \\
-0.2 & 5.000 & 5.926 & 15.000 & 29.630 & 17.778 & 11.852 & 47.407 & 41.482 \\
0 & 4.000 & 9.259 & 12.000 & 37.037 & 18.519 & 18.519 & 55.556 & 55.556 \\
0.2 & 3.333 & 13.333 & 10.000 & 44.444 & 17.778 & 26.667 & 62.222 & 71.111 \\
0.4 & 2.857 & 18.148 & 8.571 & 51.852 & 15.556 & 36.297 & 67.407 & 88.149 \\
0.6 & 2.500 & 23.704 & 7.500 & 59.259 & 11.852 & 47.407 & 71.111 & 106.667 \\
0.8 & 2.222 & 30.000 & 6.667 & 66.667 & 6.667 & 60.000 & 73.333 & 126.667 \\
\hline
\end{tabular}

Furthermore, let $\xi=-0.2$ and $\xi=0.5$ in (26), we study that the retailer's other-regarding parameter $\eta$ has effects on the supply chain system, respectively. Under both cases, we obtain Tables 3 and 4, respectively.

Obviously, Table 1 shows that Theorems 1-2 hold, and the optimal retail price and order quantity for the retailer are independent of the retailer's other-regarding parameter $\eta$, but the manufacturer's optimal wholesale price is affected by $\eta$. Table 2 shows that Theorems $3-4$ hold, and the manufacturer's other-regarding parameter $\xi$ has effects on the optimal wholesale price for the manufacturer, the optimal retail price and order quantity for the retailer. Further, we can conclude from Tables 1, 3 and 4 that: $p^{g s}<p^{g t}<p^{g g}<p^{t g}=p^{t t}=p^{t s}<p^{s g}<p^{s t}<p^{s s}$; $q^{g s}>q^{g t}>q^{g g}>q^{t g}=q^{t t}=q^{t s}>q^{s g}>q^{s t}>q^{s s} ; \Pi^{g s}>\Pi^{g t}>\Pi^{g g}>\Pi^{t g}=\Pi^{t t}=\Pi^{t s}>$ $\Pi^{s g}>\Pi^{s t}>\Pi^{s s}$.

Table 3 The supply chain system as $\xi=-0.2$

\begin{tabular}{cccccccccc}
\hline$\eta$ & $w^{*}$ & $q^{*}$ & $p^{*}$ & $\Pi_{r}^{*}$ & $U_{r}^{*}$ & $\Pi_{m}^{*}$ & $U_{m}^{*}$ & $\Pi^{*}$ & $U^{*}$ \\
\hline-0.8 & 4.778 & 3.023 & 21.000 & 27.883 & 21.164 & 8.398 & 2.822 & 36.281 & 23.986 \\
-0.6 & 4.679 & 3.750 & 18.857 & 29.595 & 23.569 & 10.044 & 4.125 & 39.639 & 27.694 \\
-0.4 & 4.679 & 4.481 & 17.250 & 30.566 & 25.765 & 12.002 & 5.889 & 42.568 & 31.654 \\
-0.2 & 4.778 & 5.208 & 16.000 & 30.671 & 27.778 & 14.468 & 8.333 & 45.139 & 36.111 \\
0 & 5.000 & 5.926 & 15.000 & 29.630 & 29.630 & 17.778 & 11.852 & 47.407 & 41.482 \\
0.2 & 5.409 & 6.629 & 14.182 & 26.819 & 31.339 & 22.600 & 17.237 & 49.419 & 48.576 \\
0.4 & 6.167 & 7.316 & 13.500 & 20.729 & 32.922 & 30.483 & 26.337 & 51.212 & 59.259 \\
0.6 & 7.769 & 7.984 & 12.923 & 6.756 & 34.392 & 46.060 & 44.709 & 52.816 & 79.101 \\
0.8 & 12.714 & 8.632 & 12.429 & -38.226 & 35.760 & 92.483 & 100.128 & 54.256 & 135.888 \\
\hline
\end{tabular}


Table 4 The supply chain system as $\xi=0.5$

\begin{tabular}{cccccccccc}
\hline$\eta$ & $w^{*}$ & $q^{*}$ & $p^{*}$ & $\Pi_{r}^{*}$ & $U_{r}^{*}$ & $\Pi_{m}^{*}$ & $U_{m}^{*}$ & $\Pi^{*}$ & $U^{*}$ \\
\hline-0.8 & 2.242 & 24.991 & 7.304 & 65.676 & 60.847 & 6.036 & 38.874 & 71.712 & 99.721 \\
-0.6 & 2.298 & 24.162 & 7.429 & 64.144 & 59.829 & 7.191 & 39.263 & 71.335 & 99.092 \\
-0.4 & 2.376 & 23.212 & 7.579 & 62.133 & 58.642 & 8.726 & 39.793 & 70.859 & 98.435 \\
-0.2 & 2.490 & 22.115 & 7.765 & 59.407 & 57.239 & 10.841 & 40.544 & 70.248 & 97.783 \\
0 & 2.667 & 20.833 & 8.000 & 55.556 & 55.556 & 13.889 & 41.667 & 69.444 & 97.222 \\
0.2 & 2.962 & 19.319 & 8.308 & 49.783 & 53.498 & 18.576 & 43.467 & 68.359 & 96.965 \\
0.4 & 3.515 & 17.506 & 8.727 & 40.316 & 50.926 & 26.524 & 46.682 & 66.840 & 97.608 \\
0.6 & 4.778 & 15.306 & 9.333 & 22.109 & 47.619 & 42.517 & 53.571 & 64.626 & 101.191 \\
0.8 & 9.143 & 12.603 & 10.286 & -28.807 & 43.210 & 90.021 & 75.617 & 61.214 & 118.827 \\
\hline
\end{tabular}

\section{Conclusions}

In this paper, we introduce the other-regarding preferences coefficients and study the impact of social preferences on supply chain performance in the price-setting newsvendor setting. It is assumed that the stochastic demand is multiplicative. The manufacturer and retailer play a Stackelberg game. We analyze the impact of the decision-maker's social preferences on the manufacturer's optimal wholesale price, the retailer's optimal retail price and order quantity, the supply chain member's profits and utilities, and the supply chain system's profits and utilities under three different cases that only the retailer, only the manufacturer and both are with social preferences. One main insight of this paper is that a supply chain will benefit when the manufacturer is generous, having concern for the total profit of the supply chain, and the retailer is spiteful, only having concern for herself.

There are several directions deserving future research. First, we study a typical channel consisting of one manufacturer and one retailer in this paper. Thus, it is more interesting to study a channel with a single manufacturer and multiple retailers. Second, we assume the manufacturer to be the Stacklberg leader in the game, but there are practical examples of large retailers (e.g., Walmart) as channel leaders. Thus, it is another interesting direction that the retailer acts as the Stacklberg leader of the channel.

\section{References}

[1] Spengler J J. Vertical integration and antitrust policy. The Journal of Political Economy, 1950, 58(4): $347-352$.

[2] Cachon G P. Supply chain coordination with contracts. The Handbook of Operations Research and Management Science: Supply Chain Management. Amsterdam, Netherlands: Kluwer Academic Publishers, 2003.

[3] Kalkanci B, Chen K Y, Erhun F. Contract complexity and performance under asymmetric demand information: An experimental evaluation. Management Science, 2011, 57(4): 689-704.

[4] Kalkanci B, Chen K Y, Erhun F. Complexity as a contract design factor: A human-to-human experimental study. Production and Operations Management, 2014, 23(2): 269-284.

[5] Rabin M. Incorporating fairness into game theory and economics. American Economic Review, 1993, 83(5): 1281-1302. 
[6] Fehr E, Schmidt K M. A theory of fairness, competition and cooperation. The Quarterly Journal of Economics, 1999, 114(3): 817-868.

[7] Charness G, Rabin M. Understanding social preferences with simple tests. The Quarterly Journal of Economics, 2002, 117(3): 817-869.

[8] Loch C H, Wu Y. Social preferences and supply chain performance: An experimental study. Management Science, 2008, 54(11): 1835-1849.

[9] Urda J, Loch C H. Social preferences and emotions as regulators of behavior in processes. Journal of Operations Management, 2013, 31: 6-23.

[10] Cui T H, Raju J S, Zhang Z J. Fairness and channel coordination. Management Science, 2007, 53(8): 1303-1314.

[11] Du S F, Du C, Liang L, et al. Supply chain coordination considering fairness concerns. Journal of Management Sciences in China, 2010, 13(11): 41-48.

[12] Katok E, Pavlov V. Fairness in supply chain contracts: A laboratory study. Journal of Operations Manangement, 2013, 31: 129-137.

[13] Liu Y, Huang Y, Luo Y, et al. How does justice matter in achieving buyer-supplier relationship performance? Journal of Operations Manangement, 2012, 30(5): 355-367.

[14] Demirag C O, Chen Y H, Li J B. Channel coordination under fairness concerns and nonlinear demand. European Journal of Operational Research, 2010, 207(3): 1321-1326.

[15] Katok E, Olsen T, Pavlov V. Wholesale pricing under mild and privately known concerns for fairness. Production and Operations Management, 2014, 23(2): 285-302.

[16] Ding C, Wang K, Lai S. Channel coordination mechanism with retailers having fairness preferences. Journal of Industrial and Management Optimization, 2013, 9(4): 967-982.

[17] Yang J, Xie J X, Deng X, et al. Cooperative advertising in a distribution channel with fairness concerns. European Journal of Operational Research, 2013, 227(2): 401-407.

[18] Wu X, Niederhoff J. Fairness in selling to the newsvendor. Production and Operations Management, 2014, 23(11): 2002-2022.

[19] Ho T H, Su X, Wu Y. Distributional and peer-induced fairness in supply chain contract design. Production and Operations Management, 2014, 23(2): 161-175.

[20] Ge Z H, Hu Q Y. Who benefits from altruism in supply chain management? American Journal of Operations Research, 2012, 2: 59-72.

[21] Shi K R, Jiang F, Ouyang Q. Altruism and pricing strategy in dual-channel supply chains. American Journal of Operations Research, 2013, 3: 402-412.

[22] Petruzzi N C, Dada M. Pricing and the newsvendor problem: A review with extensions. Operations Research, 1999, 47(2): 183-194.

[23] Wang Y Z, Li J, Shen Z J. Channel performance under consignment contract with revenue sharing. Management Science, 2004, 50(1): 33-47.

[24] Song Y Y, Ray S B, Li S L. Structural properties of buyback contracts for price-setting newsvendors. Manufacturing and Service Operations Management, 2008, 10(1): 1-18.

[25] Lariviere M A, Porteus E L. Selling to the newsvendor: An analysis of price-only contracts. Manufacturing and Service Operations Management, 2001, 3(4): 293-305. 